\title{
Impact of total variation minimization in volume rendering visualization of breast tomosynthesis data
}

\author{
A. M. Mota ${ }^{\mathrm{a}, *}$, M. J. Clarkson ${ }^{\mathrm{b}}$, P. Almeida ${ }^{\mathrm{a}}$, L. Peralta ${ }^{\mathrm{c}, \mathrm{d}}$ and N. Matela ${ }^{\mathrm{a}}$ \\ ${ }^{a}$ Instituto de Biofísica e Engenharia Biomédica, Faculdade de Ciências, Universidade de Lisboa, 1749- \\ 016 Lisboa, Portugal. \\ ${ }^{\mathrm{b}}$ Department of Medical Physics and Biomedical Engineering and the Centre for Medical Image \\ Computing, University College London, London, UK \\ ${ }^{c}$ Departamento de Física da Faculdade de Ciências da Universidade de Lisboa, Lisboa, Portugal \\ d Laboratório de Instrumentação e Física Experimental de Partículas, Lisboa, Portugal \\ *Corresponding author: \\ Ana Margarida Mota \\ Instituto de Biofísica e Engenharia Biomédica, Faculdade de Ciências da Universidade de Lisboa \\ Campo Grande, 1749-016 Lisboa, Portugal \\ ammota@fc.ul.pt
}

Concise Abstract. Total Variation (TV) minimization algorithms are one compressed sensing technique that has achieved great attention due to the virtue of decrease noise while preserve edges transitions. The purpose of this work is to solve the same TV minimization problem in DBT data, by studying two 3D filters. The obtained results were analyzed at $0^{\circ}$ and $90^{\circ}$ with a $3 \mathrm{D}$ visualization through volume rendering. The filters differ in their application. One considers a slice-by-slice optimization, sequentially traversing all slices of the data. The other considers the intensity values of adjacent slices to make this optimization on each voxel. The performance of each filter was also tested with a clinical case. The results obtained were very encouraging with a significantly increased contrast to noise ratio at $0^{\circ}$ and $90^{\circ}$ and a small reduction in blur at $90^{\circ}$ (slight reduction of the out-of-plane artifact).

Key words: Digital Breast Tomosynthesis; Total Variation minimization; volume rendering. 


\section{Abstract}

Background and Objective: Total Variation (TV) minimization algorithms have achieved great attention due to the virtue of decreasing noise while preserving edges. The purpose of this work is to implement and evaluate two TV minimization methods in 3D. Their performance is analyzed through 3D visualization of digital breast tomosynthesis (DBT) data with volume rendering.

Methods: Both filters were studied with real phantom and one clinical DBT data. One algorithm was applied sequentially to all slices and the other was applied to the entire volume at once. The suitable Lagrange multiplier used in each filter equation was studied to reach the minimum 3D TV and the maximum contrast-to-noise ratio (CNR). Imaging blur was measured at $0^{\circ}$ and $90^{\circ}$ using two disks with different diameters $(0.5 \mathrm{~mm}$ and 5.0 $\mathrm{mm}$ ) and equal thickness. The quality of unfiltered and filtered data was analyzed with volume rendering at $0^{\circ}$ and $90^{\circ}$.

Results: For phantom data, with the sequential filter, a decrease of $25 \%$ in 3D TV value and an increase of $19 \%$ and $30 \%$ in CNR at $0^{\circ}$ and $90^{\circ}$, respectively, were observed. When the filter is applied directly in 3D, TV value was reduced by $35 \%$ and an increase of $36 \%$ was achieved both for CNR at $0^{\circ}$ and $90^{\circ}$. For the smaller disk, variations of $0 \%$ in width at half maximum (FWHM) at $0^{\circ}$ and a decrease of about $2.5 \%$ for $\mathrm{FWHM}$ at $90^{\circ}$ were observed for both filters. For the larger disk, there was a $2.5 \%$ increase in FWHM at $0^{\circ}$ for both filters and a decrease of $6.28 \%$ and $1.69 \%$ in FWHM at $90^{\circ}$ with the sequential filter and the 3D filter, respectively. When applied to clinical data, the performance of each filter was consistent with that obtained with the phantom.

Conclusions: Data analysis confirmed the relevance of these methods in improving quality of DBT images. Additionally, this type of 3D visualization showed that it may 
play an important complementary role in DBT imaging. It allows to visualize all DBT data at once and to analyze properly filters applied to all the three dimensions.

\section{INTRODUCTION}

Breast cancer alone accounts for $30 \%$ of all new cancer diagnoses in women and remains the second cause of death by cancer. Besides improvements in treatment, early detection has led to a reduction of approximately $40 \%$ in the death rate from this disease over the last three decades $[1,2]$. This early detection is mostly done through screenings $[3,4]$. Approved by the Food and Drug Administration less than ten years ago [5], Digital Breast Tomosynthesis (DBT) is firmly established and is an increasingly used imaging technique for breast cancer screening and diagnosis [6-10].

So far, DBT data is displayed one slice at a time or sequentially as a continuous cine loop [11]. This procedure hampers the judgment of potential lesions such as clusters of microcalcification, which can be spread across several slices and difficult to interpret in a two dimensional (2D) image [12]. In addition, because in one DBT exam there are about 30 times more images than in 2D Digital Mammography (DM), the mean reading time doubles for DBT when compared with 2D DM examination [13-15]. Fatigue caused by the analysis of large data sets can hamper the routine functions of a radiologist. Besides, the time required for each DBT data set evaluation is crucial both in clinical and screening environments, directly influencing the number of examinations interpreted[15]. Computer Aided Detection (CAD) systems developed for DBT have presented promising preliminary results regarding the reduction of this time $[16,17]$. However, these systems are adapted to the $2 \mathrm{D}$ slice by slice visualization, which does not result immediately in a global inspection of the data. For example, in the case of clusters of microcalcifications, although decreasing the number of slices to be analyzed, it requires the radiologist to 
review multiple adjacent slices. In addition, these CAD systems, widely used in 2D DM, still present some controversy since they result in some false positives, sometimes leading to a more time consuming inspection [18].

Two other new approaches emerged in the visualization of DBT data: synthetic mammography build upon DBT data $[19,20]$ and thicker slabs obtained by combining several slices $[21,22]$. The existence of synthetic mammography is very important because it allows a fair comparison with previous DM examinations and, in some situations, it could benefit from the CAD systems developed for $2 \mathrm{D}$ application. However, as DM, synthetic mammography still has the disadvantages of a 2D visualization. For example, in the case of a dense parenchyma pattern or microcalcification clusters, its clinical value is limited due to tissue overlapping (it is used in combination with DBT slice-by-slice) [23]. On the other hand, the construction of slabs has demonstrated good results in terms of reducing time and false positives but originates a lower sensitivity [22].

A different type of visualization may play an important complementary role in breast cancer diagnosis [24]. Three dimensional (3D) volume rendering is the process of creating realistic computer-generated images of a 3D scene, yielding an improved depth perception [25]. 3D volume rendering proved to be useful in tomographic medical imaging modalities such as Computed Tomography (CT), Magnetic Resonance Imaging or Positron Emission Tomography [26-28]. There are some works mentioning a few aspects of 3D volume rendering for DBT [29-31] and its importance to detect clusters of microcalcifications [32]. As in $\mathrm{CT}$, the $3 \mathrm{D}$ visualization integration in a $2 \mathrm{D}$ reading can be seen as a problem solver in some situations (for example, the necessity to better understand the shape of a cluster of microcalcifications) [33]. 
In DBT, a small number of low-dose projections acquired within a limited angular range are reconstructed to produce $3 \mathrm{D}$ data of breast tissue. Both the low dose per projection and the angular limit raise some complications in the reconstruction of DBT data. The first results in high noise level in the reconstructed images, while the second restricts the spatial resolution in the direction perpendicular to the detector plane (the $\mathrm{z}$ direction), resulting in out-of-plane blur. Visible in 2D slice-by-slice visualization, the out-of-plane artifact becomes even more evident in 3D volume rendering. Reducing noise without blurring or decreasing details and edge definition is a challenge in image processing. Total Variation (TV) minimization algorithms are efficient in reducing noise while preserving edges. For this reason, studies applying TV minimization to DBT data have grown significantly. Most works focus on the use of these algorithms in iterative reconstruction [34-41], with a few focusing on post-processing [42-44]. The Lagrange multiplier $(\lambda)$ is an important parameter in TV minimization [42] since it controls image regularization, giving a trade-off between removing noise and preserving edges. For this reason, to find the appropriate $\lambda$ value is extremely important in this type of regularization.

In this paper, two TV minimization algorithms based on [42] and [44] were applied to DBT reconstructed data and their impact on the volume rendering visualization was analyzed. The first is a 2D TV minimization filter applied to a single slice [42], while the second is a 3D TV minimization filter applied to the entire volume and analyzed through a preliminary study in a 2D way (in-plane analysis) [44]. In this work, the 2D TV minimization filter was applied sequentially to all phantom slices and both the results, from 2D and 3D filters, were analyzed based on volumetric rendering of DBT phantom data, considering their performance in z-direction. Despite the limited resolution in $\mathrm{z}$, volume rendering is useful to visualize all DBT data at once (with particular emphasis on high intensity features, such as calcifications). In addition, as it results in a truly 3D 
visualization, it is an appropriate way to analyze filters applied to all three dimensions. Since the Lagrange multiplier is a decisive parameter in TV minimization, the suitable $\lambda$ to be used was studied (in a first phase to reach the minimum 3D TV of the data and in a second stage to achieve the maximum contrast to noise value in a specific region). Quantitative and visual analyses were conducted between unfiltered and filtered rendered images and also between the two different TV minimization filters. The proposed algorithms were also tested with one clinical DBT data set.

\section{METHODS}

\section{1. Data acquisition and reconstruction}

To mimic the breast tissue, an acrylic phantom made by us was scanned with a Siemens MAMMOMAT Inspiration system (Siemens AG, Healthcare Sector, Erlangen, Germany) installed in a clinical facility (Hospital da Luz S.A., Lisbon, Portugal). The phantom was acquired with $28 \mathrm{kVp}$ and $47 \mathrm{mAs}$. To simulate high-density lesions, the phantom contains two columns of aluminum disks of different diameters and $1 \mathrm{~mm}$ thickness (Fig. 1). For this study the first column was considered.

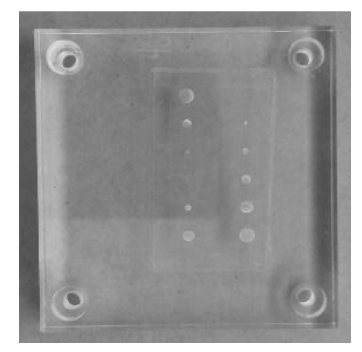

Fig. 1. Acrylic phantom simulating breast tissue and lesions of high attenuation (aluminum disks of different diameters and $1 \mathrm{~mm}$ thickness). Diameter of the first column disks (top to bottom): $5 \mathrm{~mm}, 3 \mathrm{~mm}, 1 \mathrm{~mm}, 0.5 \mathrm{~mm}, 2 \mathrm{~mm}$ and 4 $\mathrm{mm}$, respectively.

Additionally, one clinical DBT data set with a large calcification of an anonymous patient was selected from the clinical facility database. This clinical data set was acquired with $26 \mathrm{kVp}$ and $34 \mathrm{mAs}$. 
The acquired data were reconstructed with the manufacturer algorithm which uses Filtered Back Projection (FBP) with some post-processing to reduce artifacts and image blurring [45]. The reconstructions have voxel sizes of $0.085 \times 0.085 \times 1.0 \mathrm{~mm}^{3}$. The algorithms under study were implemented using MATLAB R2016b and run on a computer containing 4 Intel® Xeon(R) CPU E5620 @ 2.40 GHz.

\section{2. Data visualization}

In volume rendering, changing the azimuth of a camera rotates its position around the focal point [46]. In this case, two different angle position were used to visualize the DBT data: $0^{\circ}$ and $90^{\circ}$. The first corresponds to the visualization parallel to the detector plate (i.e. the projection is made on the xy planes along $z$ ) and the second is the visualization perpendicular to the detector plate (i.e. projection is made on the $\mathrm{xz}$ planes along y).

The visualization software was developed in $\mathrm{C}++$ using the Visualization Toolkit library (VTK) version 7.1.0. [46, 47]. For a better visualization quality, voxels were made isotropic (with dimensions $0.085 \times 0.085 \times 0.085 \mathrm{~mm}^{3}$ ) using the Lanczos function available in VTK (used by default) [46, 48].

The methodologies in study were analyzed using 3D volume rendering visualization with a ray casting algorithm and compositing technique. Different rendering parameters yield different images. For this reason, the parameters were fixed for all situations so that a correct comparison could be made.

\section{3. Image analysis}

For image analysis, quantitative and qualitative comparisons were performed between unfiltered and filtered rendered images at $0^{\circ}$ and $90^{\circ}$. For phantom quantitative 
analysis, two figures of merit were used: Contrast to noise ratio (CNR) and full width at half maximum (FWHM) of a Gaussian curve fitted to the profile of two disks $-0.5 \mathrm{~mm}$ and $5.0 \mathrm{~mm}$. For CNR, a region of interest (ROI) over the $5 \mathrm{~mm}$ disk and four other ROIs over the surrounding background were drawn. The CNR was calculated with Eq. (1), where $\mu_{\mathrm{F}}$ and $\mu_{\mathrm{B}}$ stand for the mean pixel values in the ROI over the selected feature (5.0 mm disk) and the background, respectively; and $\sigma_{\mathrm{B}}$ stands for the mean of standard deviations in the background ROIs.

$$
C N R=\frac{\mu_{F}-\mu_{B}}{\sigma_{B}}
$$

At $0^{\circ}$, the FWHM of the $0.5 \mathrm{~mm}$ disk was considered as an indicator of spatial resolution. At $90^{\circ}$, the FWHM of the $0.5 \mathrm{~mm}$ and $5 \mathrm{~mm}$ disks were considered as indicators of the blur in the $\mathrm{z}$ direction.

\section{4. Formulation of TV minimization problem}

The 3D TV values were calculated according to Eq. (2) where $u_{i, j, k}$ is the intensity value of $\operatorname{voxel}(i, j, k)$, with $i=\{1, \ldots, m\}, j=\{1, \ldots, n\}, k=\{1, \ldots, p\}$ and $m \times n \times$ $p$ the data dimensions. $\Delta_{x}, \Delta_{y}$ and $\Delta_{z}$ are discretizations of the horizontal (x), vertical (y) and perpendicular (z) derivatives, respectively.

$$
T V_{3 D}(u)=\sum_{i=1}^{m} \sum_{j=1}^{n} \sum_{k=1}^{p} \sqrt{\left(\Delta_{x} u_{i, j, k}\right)^{2}+\left(\Delta_{y} u_{i, j, k}\right)^{2}+\left(\Delta_{z} u_{i, j, k}\right)^{2}}
$$

The unconstrained TV minimization problem here addressed is based on ROF model [49] for Poisson noise [50, 51] and can be formulated as in Eq. (3). $f$ and $u$ are the original and denoised data, respectively, $T V(u)$ is the TV of denoised data and $\lambda$ is the Lagrange multiplier, also called the regularization parameter. 


$$
\min _{u}\{T V(u)+\lambda(u-f \ln u)\}
$$

In Eq. (3), the first term corresponds to the regularization term (TV function) and the second one relates with the assumed noise model (fidelity term). In this way, $\lambda$ controls data regularization, between removing noise and preserving information.

The problem represented in Eq. (3) can be solved through its Euler-Lagrange equation in finite data domain $\Omega$ ( $\partial u / \partial n=0$ on $\partial \Omega$, with $n$ relative to noise). Eq. (4) stands for the 2D TV minimization filter and was obtained based on discretization of the corresponding Euler-Lagrange equation as in [42].

$$
0=\frac{\lambda}{u}(u-f)-\left[\Delta_{x}^{-} \frac{\Delta_{x}^{+} u_{i, j}}{\sqrt{\varepsilon^{2}+\left(\Delta_{x}^{+} u_{i, j}\right)^{2}+\left(\Delta_{y}^{0} u_{i, j}\right)^{2}}}+\Delta_{y}^{-} \frac{\Delta_{y}^{+} u_{i, j}}{\sqrt{\varepsilon^{2}+\left(\Delta_{x}^{0} u_{i, j}\right)^{2}+\left(\Delta_{y}^{+} u_{i, j}\right)^{2}}}\right] \text { (4) }
$$

$u_{i, j}$ is the intensity value of voxel $(i, j)$ at slice $k$. Each voxel to be denoised only depends on the intensity values of the surrounding voxels in the same slice (Fig. 2 (a)). As this is a 3D study, Eq.(4) was applied sequentially to all slices $(k=\{1, \ldots, p\})$.

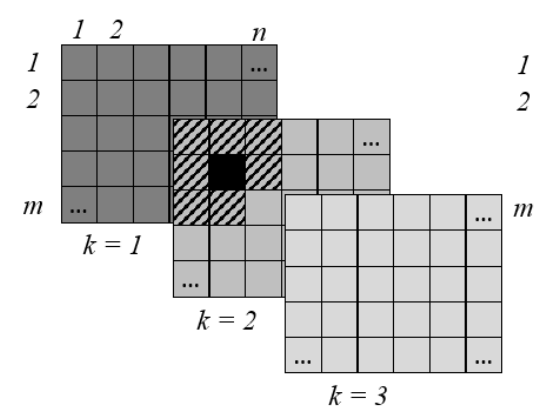

(a)

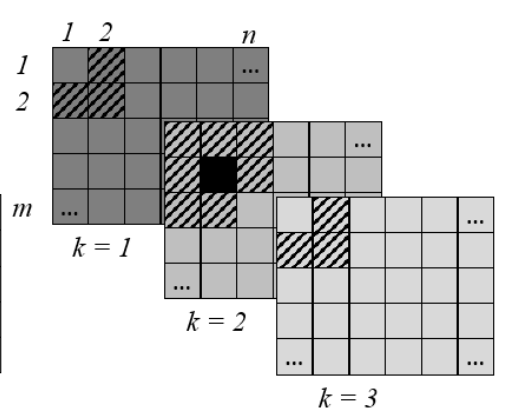

(b)

Fig. 2. Illustrative scheme of the application of each filter in slice number two. (a) 2D TV minimization filter applied to all slices, one slice at a time. (b) 3D TV minimization filter.

In each slice, each voxel to be denoised with the 3D TV minimization filter is influenced by the intensity values of the surrounding voxels, including voxels in the neighboring slices. (Fig. 2 (b)). In this work, the discrete equation (Eq. (5)) which corresponds to the Euler-Lagrange solution of the 3D TV minimization problem, presents some differences in relation to [44], specifically in the discretization of derivatives. These 
minor changes were achieved after some empirical studies where it was found that these combinations allow for a better balance between differences.

$$
\begin{aligned}
0=\frac{\lambda}{u}(u-f)-\left[\Delta_{x}^{-} \frac{\Delta_{x}^{+} u_{i, j, k}}{\sqrt{\varepsilon^{2}+\left(\Delta_{x}^{+} u_{i, j, k}\right)^{2}+\left(\Delta_{y}^{0} u_{i, j, k}\right)^{2}+\left(\Delta_{z}^{0} u_{i, j, k}\right)^{2}}}\right. \\
+\Delta_{y}^{-} \frac{\Delta_{y}^{+} u_{i, j, k}}{\sqrt{\varepsilon^{2}+\left(\Delta_{x}^{0} u_{i, j, k}\right)^{2}+\left(\Delta_{y}^{+} u_{i, j, k}\right)^{2}+\left(\Delta_{z}^{0} u_{i, j, k}\right)^{2}}} \\
\left.+\Delta_{z}^{-} \frac{\Delta_{z}^{+} u_{i, j, k}}{\sqrt{\varepsilon^{2}+\left(\Delta_{x}^{0} u_{i, j, k}\right)^{2}+\left(\Delta_{y}^{0} u_{i, j, k}\right)^{2}+\left(\Delta_{z}^{+} u_{i, j, k}\right)^{2}}}\right]
\end{aligned}
$$

In Eq. (4) and Eq. (5), $\Delta_{x}^{+}, \Delta_{x}^{-}, \Delta_{y}^{+}, \Delta_{y}^{-}, \Delta_{z}^{+}$and $\Delta_{z}^{-}$, denote forward (+) and backward (-) one-sided differences in $x, y$ and $z$ directions, respectively; $\Delta_{x}^{0}, \Delta_{y}^{0}$ and $\Delta_{z}^{0}$ indicate central difference in $\mathrm{x}, \mathrm{y}$ and $\mathrm{z}$ directions, respectively; and $\varepsilon>0$ is a small parameter introduced to remove the derivative singularity when $u$ is locally constant.

These formulations are valid only for interior points, i.e., voxel $(i, j, k)$ with $i=\{2, \ldots, m-1\}, j=\{2, \ldots, n-1\}$ and $k=\{2, \ldots, p-1\}$, excluding borders. For the $2 \mathrm{D}$ problem (Eq.(4)), boundary conditions were defined as in [52]. For the 3D algorithm (Eq. (5)), boundary conditions were defined as:

$$
\left\{\begin{array}{l}
u_{1, j, k}=u_{2, j, k} \text { and } u_{m, j, k}=u_{m-1, j, k} \text { for } j=\{2, \ldots, n-1\} \text { and } k=\{2, \ldots, p-1\} ; \\
u_{i, 1, k}=u_{i, 2, k} \text { and } u_{i, n, k}=u_{i, n-1, k} \text { for } i=\{2, \ldots, m-1\} \text { and } k=\{2, \ldots, p-1\} \\
u_{i, j, 1}=u_{i, j, 2} \text { and } u_{i, j, p}=u_{i, j, p-1} \text { for } i=\{2, \ldots, m-1\} \text { and } j=\{2, \ldots, n-1\} \\
u_{1,1,1}=u_{2,2,1}, u_{1, n, 1}=u_{2, n-1,1}, u_{m, 1,1}=u_{m-1,2,1}, u_{m, n, 1}=u_{m-1, n-1,1}, \\
u_{1,1, p}=u_{2,2, p}, u_{1, n, p}=u_{2, n-1, p}, u_{m, 1, p}=u_{m-1,2, p}, u_{m, n, p}=u_{m-1, n-1, p}
\end{array}\right.
$$


The main differences between the previously studied algorithms $[42,44]$ and those applied in this study are summarized in the Table 1.

Table 1. Summary of the differences between the previous studies using the mentioned TV minimization algorithms $[42,44]$ and the studies developed in this work.

\begin{tabular}{|c|c|c|c|c|}
\hline & Ref. [42] & $\begin{array}{l}\text { TV min in all } \\
\text { slices } \\
\text { (Fig. } 2 \text { (a)) }\end{array}$ & Ref. [44] & $\begin{array}{l}\text { TV min } \\
\text { in the volume } \\
\text { (Fig. } 2(\mathrm{~b}))\end{array}$ \\
\hline Phantom & $\begin{array}{l}\text { Mammographic } \\
\text { Accreditation } \\
\text { Phantom Model } \\
156\end{array}$ & $\begin{array}{l}\text { Acrylic phantom } \\
\text { simulating breast } \\
\text { tissue and lesions } \\
\text { of high attenuation }\end{array}$ & $\begin{array}{c}\text { Mammographic } \\
\text { Accreditation } \\
\text { Phantom Model } \\
156\end{array}$ & $\begin{array}{c}\text { Acrylic phantom } \\
\text { simulating breast } \\
\text { tissue and lesions } \\
\text { of high } \\
\text { attenuation }\end{array}$ \\
\hline $\begin{array}{l}\text { Reconstructed } \\
\text { voxel }\left(\mathrm{mm}^{3}\right)\end{array}$ & $0.34 \times 0.34 \times 1.0$ & $\begin{array}{c}0.085 \times 0.085 \times \\
1.0 \\
\end{array}$ & $0.34 \times 0.34 \times 1.0$ & $\begin{array}{c}0.085 \times 0.085 \times \\
1.0\end{array}$ \\
\hline $\begin{array}{l}\text { Reconstruction } \\
\text { algorithm }\end{array}$ & $\begin{array}{c}\text { Algebraic } \\
\text { Reconstruction } \\
\text { Technique } \\
\end{array}$ & $\begin{array}{c}\text { Filtered } \\
\text { Backprojection }\end{array}$ & $\begin{array}{c}\text { Algebraic } \\
\text { Reconstruction } \\
\text { Technique } \\
\end{array}$ & $\begin{array}{c}\text { Filtered } \\
\text { Backprojection }\end{array}$ \\
\hline Implementation & $\begin{array}{l}\text { Ref. [42] was app } \\
\text { of interest whil } \\
\text { algorithm of Fig. } \\
\text { all slices sequent }\end{array}$ & $\begin{array}{l}\text { ied to a single slice } \\
\text { in this study, the } \\
\text { (a) was applied to } \\
\text { ally (is a 3D filter). }\end{array}$ & \multicolumn{2}{|c|}{$\begin{array}{l}\text { A different combination of discrete } \\
\text { derivatives was used in Eq. (5). In the } \\
\text { square root, the combination in this } \\
\text { study was: }(+, 0,0),(0,+, 0) \text { and }(0,0,+) \\
\text { instead of }(+, 0,-),(-,+, 0) \text { and }(0,-,+) \\
\text { used in the previous study [44]. With } \\
\text { this combination there was an } \\
\text { improvement of about } 0.3 \% \text { in 3D TV } \\
\text { minimization. }\end{array}$} \\
\hline
\end{tabular}

\section{5. Optimization of TV minimization filter}

Filters' performance directly depend on the regularization parameter $(\lambda)$ used in Eqs. (4) and (5). $\lambda$ allows to control the weight between the two terms in Eq. (3): Regularization Vs. Fidelity.

\section{5. 1. Comprehensive study of $\lambda$}

There is one $\lambda$ value for which the minimum 3D TV is obtained, while maintaining the fidelity of data. Although both the algorithms take into account the entire data (not only one slice), their application is different (Fig. 2). For this reason, minimum 3D TV values of filtered data will be different, and so the respective $\lambda . \lambda$ values ranging between $[10,250]$ were applied and the results obtained for both filters were analyzed. 
The validity of $2 \mathrm{D}$ filter when applied to one single slice has already been carefully studied in [42] while preliminary results achieved with the 3D filter were presented in [44]. The aim of this comprehensive study was to validate both the algorithms under the modified conditions: the 2D algorithm considering all slices and the 3D filter after the introduced alterations. Additionally, it was also useful to find $\lambda$ reference values for the next section.

\section{5. 2. $\lambda$ Vs. CNR $\max \left(90^{\circ}\right)$}

The $\lambda$ value which allows the greatest minimization of $3 \mathrm{D} T \mathrm{TV}$ of the entire data may differ from the value which generates reduction of local blur or improvement of local CNR. In addition, as there is a minimization of TV and therefore noise and variability reduction, the impact of these filters in z-direction should be studied through image quality at $90^{\circ}$. For this reason, based on $\lambda$ values responsible for the greatest minimization of $3 \mathrm{D} \mathrm{TV}$, a detailed study about $\mathrm{CNR}$ at $90^{\circ}$ as a function of $\lambda$ was conducted. Images generated by $\lambda$ values allowing the highest $\mathrm{CNR}$ at $90^{\circ}$ have been analyzed.

\section{6. DBT clinical data}

For the clinical quantitative analysis, CNR and FWHM were also obtained at $0^{\circ}$ and $90^{\circ}$. CNR was calculated as in Eq. (1), with the feature of interest being the biggest calcification. The FWHM was obtained with a Gaussian curve fitted to the profile of the same calcification. It is known that the calcification diameter is approximately 3.6-3.8 $\mathrm{mm}$ at $0^{\circ}$ (xy planes) and $8-11 \mathrm{~mm}$ in $90^{\circ}$ (yz planes), respectively. As in phantom measures, $\mathrm{FWHM}$ value at $90^{\circ}$ was considered as an indicator of the blur in $\mathrm{z}$ direction.

\section{RESULTS}


Both filters were applied to the phantom with original dimensions of $1330 \times 1460 \times 42$ and voxel size $0.085 \times 0.085 \times 1.0 \mathrm{~mm}^{3} .2 \mathrm{D}$ TV minimization algorithm took approximately 17 seconds to cover all slices and 3D TV minimization was done in approximately 28 seconds. The results obtained are shown in Secs. 3.A-3.E.

\subsection{Comprehensive study of $\lambda$ Vs. 3D TV values}

The results for 3D TV values (calculated using Eq. (2)) obtained with $\lambda$ ranging between 10 and 250 (with a fixed step-size of 10) are presented in Fig. 3 and Fig. 4 for the 2D TV minimization in all slices and 3D TV minimization, respectively. For more detailed information, the $\lambda$ value was varied with a step-size of 1 and the first filter was then applied with $\lambda$ between 188 and 208 (Fig. 3-right) and the second one with $\lambda$ ranging from 215 and 235 (Fig. 4-right).

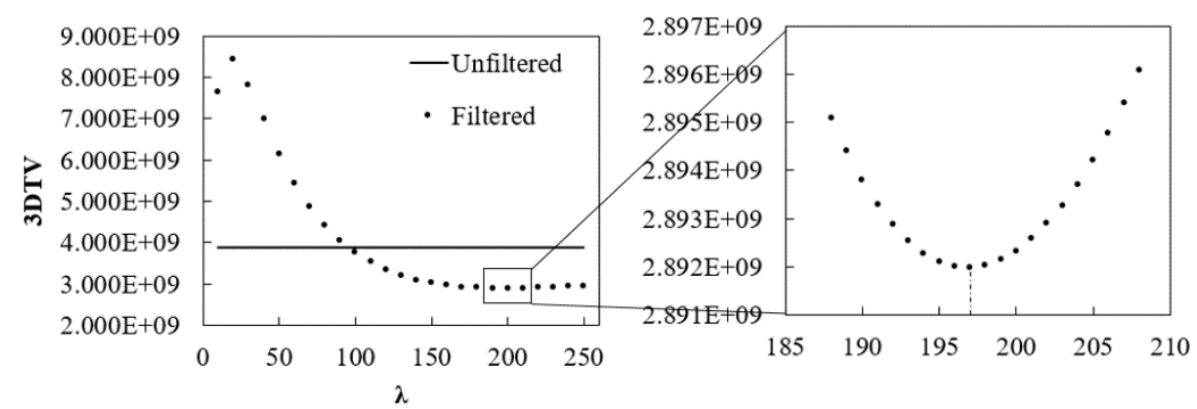

Fig. 3. 3D TV values for unfiltered and filtered phantom data, with minimization of 2D TV in all slices, plotted as a function of $\lambda$. Right: zoom in on $\lambda$ range where $3 \mathrm{D}$ TV is minimum.

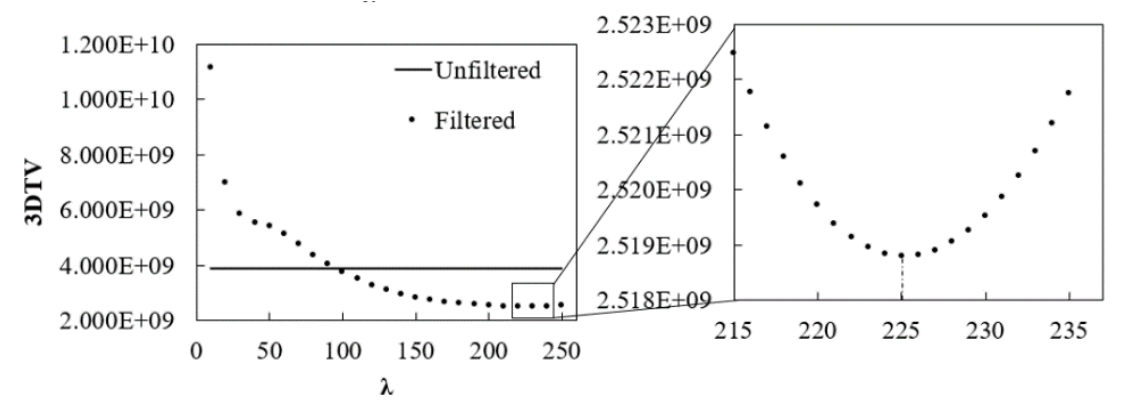

Fig. 4. 3D TV values for unfiltered and filtered phantom data, with minimization of 3D TV, plotted as a function of $\lambda$. Right: zoom in on $\lambda$ range where $3 \mathrm{D}$ TV is minimum. 
The 3D TV value calculated for the unfiltered data and minimum 3D TV values obtained with each filter (and respective $\lambda$ ) are presented in Table 2.

Table 2. Summary of the results obtained for the 3D TV values of unfiltered and filtered phantom data with the two applications (minimization of 2D TV in all slices and minimization of 3D TV). It is also presented the variation in percentage between the unfiltered and filtered values.

\begin{tabular}{lccc}
\hline & TV3D & $\lambda$ & $\Delta(\%)$ \\
\hline Unfiltered & $3.885 \mathrm{E}+09$ & & \\
\hline Filtered min2DTV all slices & $2.892 \mathrm{E}+09$ & 197 & -25.57 \\
Filtered min3DTV & $2.519 \mathrm{E}+09$ & 225 & -35.17 \\
\hline
\end{tabular}

\section{2. $\lambda$ Vs. CNRmax $\left(90^{\circ}\right)$}

From Table $2, \lambda(\min 2 D T V$-allslices $)=197$ and $\lambda(\min 3 D T V)=225$ allow the lowest 3D TV value for each filter. For this reason, CNR calculated values in the $5 \mathrm{~mm}$ disk at $90^{\circ}$ in the phantom filtered data as a function of $\lambda$ values ranging between $[155,245]$ are presented in Fig. 5.
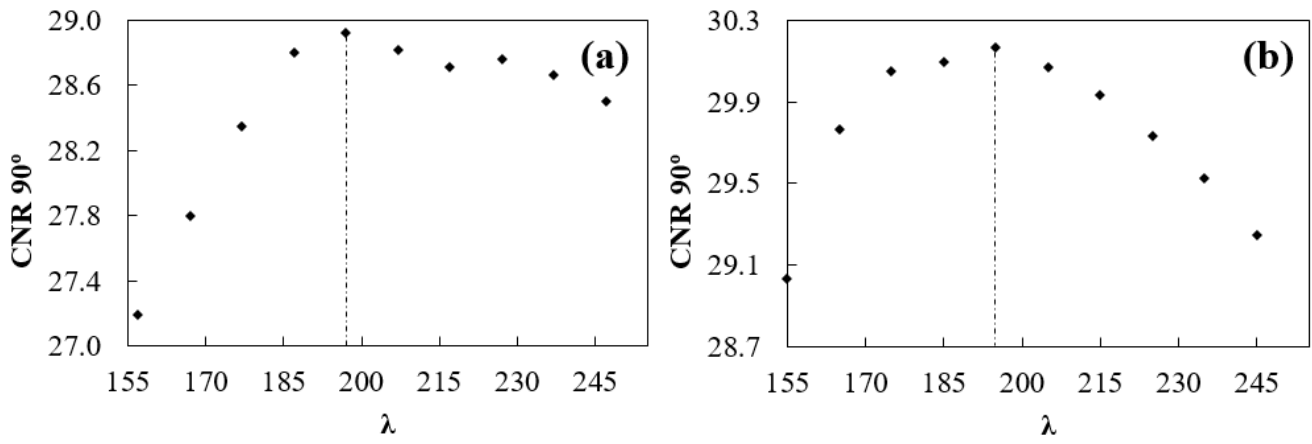

Fig. 5. Values of CNR at $90^{\circ}$ obtained with 2D TV minimization algorithm applied to all slices (a) and 3D TV minimization algorithm (b) as a function of $\lambda$.

\subsection{Phantom analysis}

From Fig. 5 (a), for the $2 \mathrm{D}$ TV minimization algorithm, $\lambda=197$ was the value which allows the maximum CNR at $90^{\circ}$. On the other hand, $\lambda=195$ was the best choice for the 3D TV minimization algorithm (Fig. 5 (b)). Table 3 summarizes the quantitative results obtained for the filtered data at $90^{\circ}$ using these $\lambda$ values. 
in all slices and $\lambda=195$ for the 3D TV minimization). It is also presented the variation in percentage between the unfiltered and filtered values.

\begin{tabular}{lcccccc}
\hline & \multicolumn{9}{c}{$90^{\circ}$} \\
\hline & $\mathrm{CNR}_{\max }$ & \multicolumn{3}{c}{$\begin{array}{c}\mathrm{FWHM}_{0.5 \mathrm{~mm}} \\
(\mathrm{~mm})\end{array}$} & \multicolumn{3}{c}{$\begin{array}{c}\mathrm{FWHM}_{5.0 \mathrm{~mm}} \\
(\mathrm{~mm})\end{array}$} \\
\hline Unfiltered & 22.106 & $\Delta(\%)$ & 2.594 & $\Delta(\%)$ & 3.100 & $\Delta(\%)$ \\
\hline $\begin{array}{l}\text { Filtered min2DTV } \\
\text { all slices }\end{array}$ & 28.924 & 30.84 & 2.517 & -2.97 & 2.906 & -6.28 \\
Filtered min3DTV & 30.167 & 36.47 & 2.533 & -2.36 & 3.048 & -1.69 \\
\hline
\end{tabular}

As DBT data and algorithms are 3D, besides analysis in z-direction, it is important to also ensure quality at $0^{\circ}$. In this way, the quantitative values obtained in the $x y$ reconstruction plane are presented in Table 4.

Table 4. Results obtained for CNR, FWHM ${ }_{0.5 \mathrm{~mm}}$ and FWHM $5.0 \mathrm{~mm}$ values of unfiltered and filtered phantom data at $0^{\circ}$. Each filtered data was obtained using $\lambda$ corresponding to the maximum CNR at $90^{\circ}(\lambda=197$ for the $2 \mathrm{D}$ TV minimization in all slices and $\lambda=195$ for the $3 \mathrm{D}$ TV minimization). It is also presented the variation in percentage between the unfiltered and filtered values.

\begin{tabular}{lcccccc}
\hline & \multicolumn{9}{c}{$0^{\circ}$} \\
\hline & CNR $_{\max }$ & \multicolumn{3}{c}{$\begin{array}{c}\text { FWHM }_{0.5 \mathrm{~mm}} \\
(\mathrm{~mm})\end{array}$} & $\begin{array}{c}\text { FWHM }_{5.0 \mathrm{~mm}} \\
(\mathrm{~mm})\end{array}$ & \\
\hline Unfiltered & 17.427 & $\Delta(\%)$ & 0.4957 & $\Delta(\%)$ & 4.479 & $\Delta(\%)$ \\
\hline $\begin{array}{l}\text { Filtered min2DTV } \\
\text { all slices }\end{array}$ & 20.822 & 19.48 & 0.4956 & -0.02 & 4.595 & 2.58 \\
Filtered min3DTV & 23.761 & 36.35 & 0.4962 & 0.09 & 4.598 & 2.65 \\
\hline
\end{tabular}

Images of results achieved with volume rendering at $0^{\circ}$ of the $0.5 \mathrm{~mm}$ and $5 \mathrm{~mm}$ disks are presented in Fig. 6 and images at $90^{\circ}$ are shown in Fig. 7. In both Fig. 6 and Fig. 7, the first column (a1 and a2) represent unfiltered disks, the second column (b1 and b2) presents filtered disks with 2D TV minimization in all slices, and the last column (c1 and c2) shows the results obtained with 3D TV filter. 

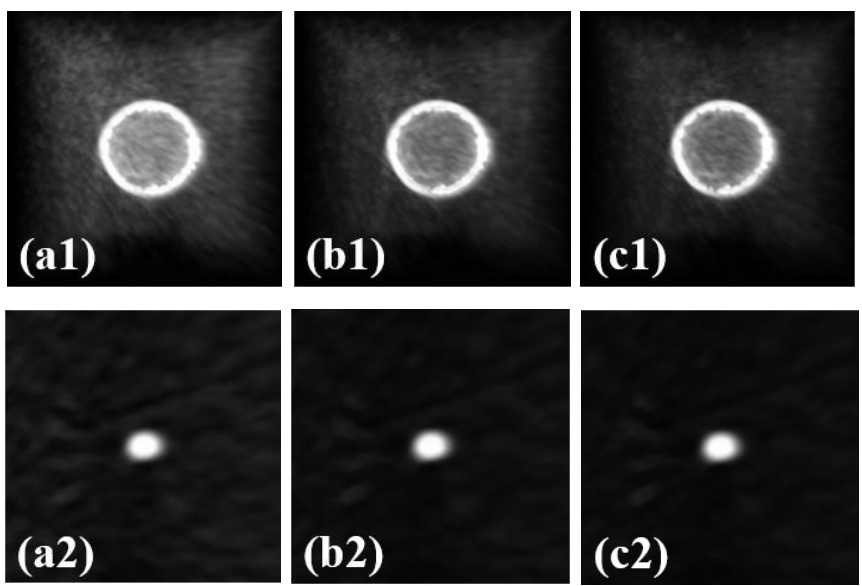

Fig. 6. Volume rendering images at $0^{\circ}$ for $5 \mathrm{~mm}$ disk (top row) and $0.5 \mathrm{~mm}$ disk (bottom row) obtained for the unfiltered (a1 and a2) and filtered data with 2D TV minimization filter applied to all slices $-\lambda=197$ (b1 and b2) and 3D TV minimization filter $-\lambda=195(\mathrm{c} 1$ and $\mathrm{c} 2$ ).
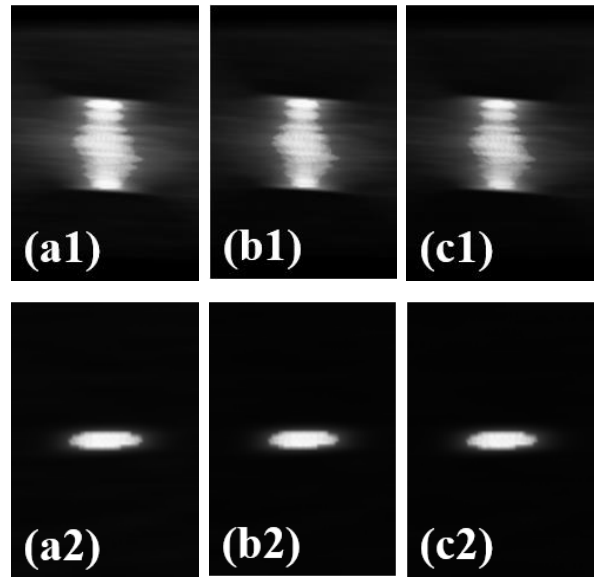

Fig. 7. Volume rendering images at $90^{\circ}$ for $5 \mathrm{~mm}$ disk (top row) and $0.5 \mathrm{~mm}$ disk (bottom row) obtained for the unfiltered (a1 and a2) and filtered data with 2D TV minimization filter applied to all slices $-\lambda=197$ (b1 and b2) and 3D TV minimization filter $-\lambda=195$ (c1 and $\mathrm{c} 2$ ).

\subsection{Clinical data analysis}

The results for 3D TV values obtained for clinical data with $\lambda$ ranging between 10 and 200 are presented in Fig. 8 and Fig. 9 for the 2D TV minimization in all slices and 3D TV minimization, respectively. For further analysis, 3D TV values of filtered clinical data were obtained with $\lambda$ ranging from 124 and 144 for the first filter (Fig. 8-right) and $\lambda$ between 135 and 155 for the second (Fig. 9-right). 


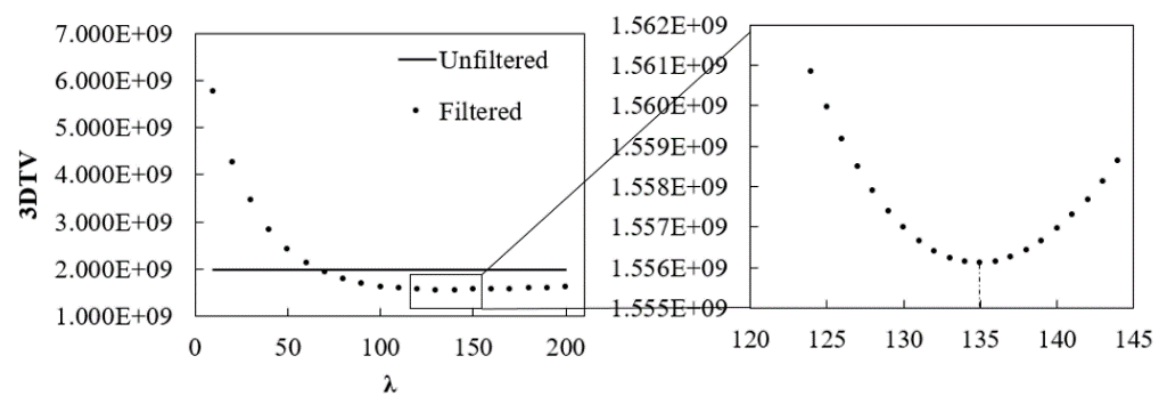

Fig. 8. 3D TV values for unfiltered and filtered clinical data, with minimization of 2D TV in all slices, plotted as a function of $\lambda$. Right: zoom in on $\lambda$ range where $3 \mathrm{D} T \mathrm{TV}$ is minimum.

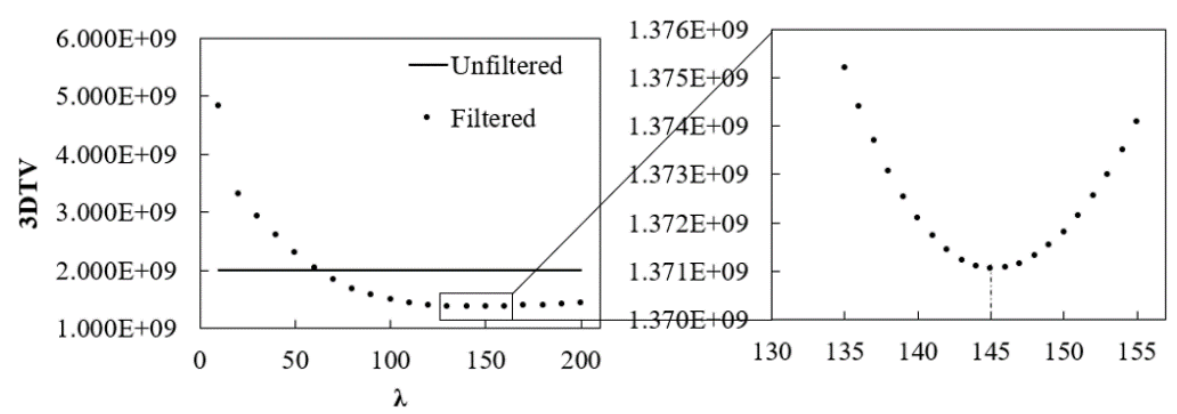

Fig. 9. 3D TV values for unfiltered and filtered clinical data, with minimization of 3D TV, plotted as a function of $\lambda$. Right: zoom in on $\lambda$ range where $3 \mathrm{D}$ TV is minimum.

The 3D TV value calculated for the unfiltered clinical data and the minimum 3D TV values obtained after the application of each filter (and respective $\lambda$ ) are summarized in Table 5.

Table 5. Summary of the results obtained for the 3D TV values of unfiltered and filtered clinical data with the two applications (minimization of 2D TV in all slices and minimization of 3D TV). It is also presented the variation in percentage between the unfiltered and filtered values.

\begin{tabular}{lccc}
\hline & TV3D & $\lambda$ & $\Delta(\%)$ \\
\hline Unfiltered & $1.995 \mathrm{E}+09$ & & \\
\hline Filtered min2DTV all slices & $1.556 \mathrm{E}+09$ & 135 & -22.02 \\
Filtered min3DTV & $1.371 \mathrm{E}+09$ & 145 & -31.29 \\
\hline
\end{tabular}

In Table 6 are presented the CNR and FWHM values measured, at $0^{\circ}$ and $90^{\circ}$, before and after the application of each filter using the respective $\lambda$ value that allowed to obtain the minimum 3D TV value. In Fig. 10, volume rendering of the results obtained with clinical data at $0^{\circ}$ and $90^{\circ}$ are illustrated.

Table 6. Results obtained for CNR and FWHM Calc values of unfiltered and filtered clinical data at $0^{\circ}$ and $90^{\circ}$. Each filtered data was obtained using $\lambda$ corresponding to the minimum 3D TV ( $\lambda=135$ for the $2 \mathrm{D}$ TV minimization in all 
slices and $\lambda=145$ for the $3 \mathrm{D}$ TV minimization). It is also presented the variation in percentage between the unfiltered and filtered values.

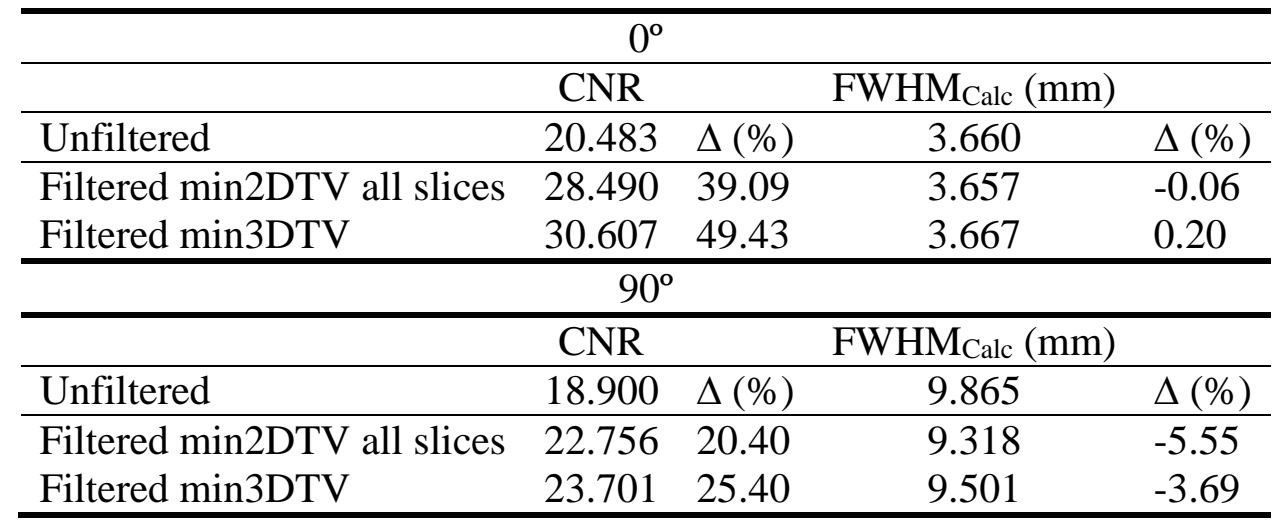
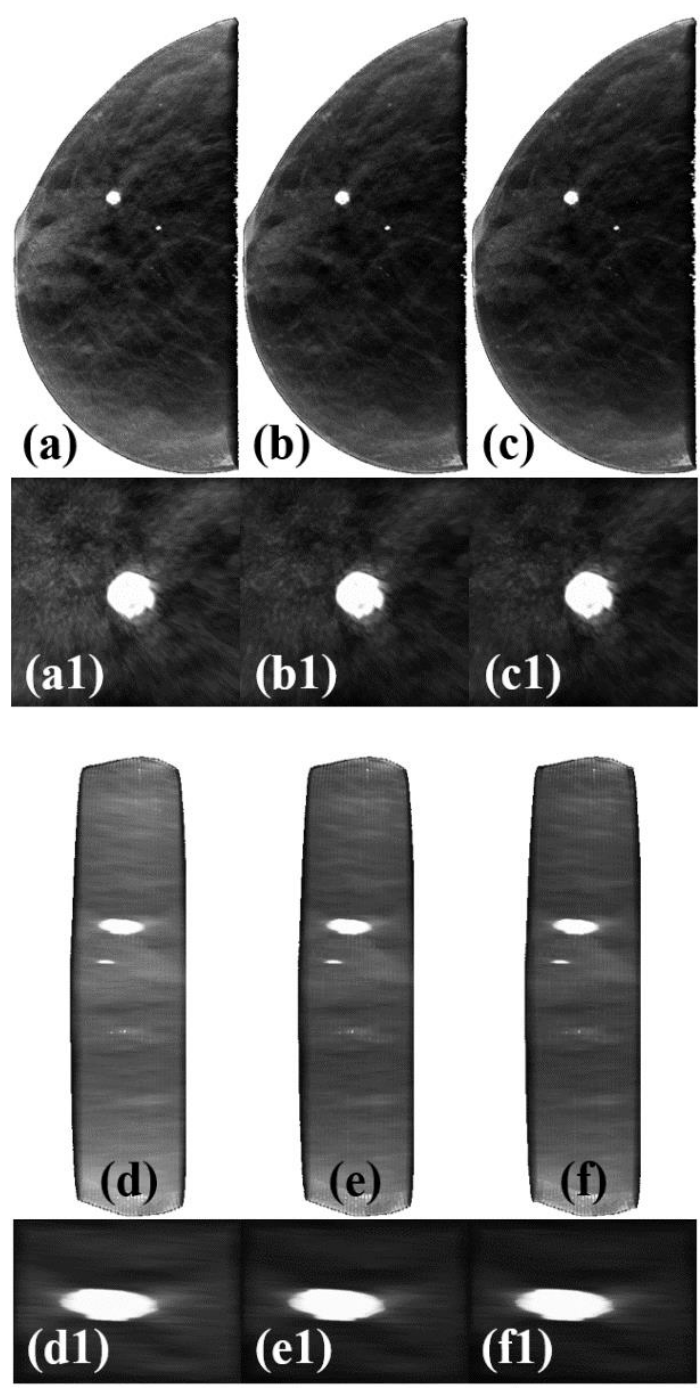

Fig. 10. Volume rendering at $0^{\circ}\left(\mathrm{a}, \mathrm{b}\right.$ and $\mathrm{c}$ ) and $90^{\circ}$ (d, e and f) of clinical data. (a1, b1, c1, d1, e1 and f1) represent the zoom in of the biggest calcification. Unfiltered data: a (a1) and d (d1); filtered data with 2D TV minimization: b (b1) and e (e1); and data obtained with 3D TV minimization filter: $\mathrm{c}(\mathrm{c} 1)$ and $\mathrm{f}(\mathrm{f} 1)$. 


\section{DISCUSSION}

The application of this type of filters after reconstruction is a straightforward approach and it is not time-consuming, unlike iterative methods. In addition, these filters are applied to all dimensions of DBT data and the visualization of obtained results through $3 \mathrm{D}$ volume rendering allows a better analysis of the effects in all directions. Here, two TV minimization filters were optimized considering the three dimensions and our study focused on: the $\lambda$ value that solves the $3 \mathrm{D}$ TV minimization problem, validating the algorithms and the $\lambda$ value which gives the maximum CNR in a high intensity region at $90^{\circ}$ (without compromising or even improving the z-blur).

Considering the results obtained with the phantom, we observe that both filters had more influence in CNR values than on FWHM, at $0^{\circ}$ and $90^{\circ}$. Regardless of the filter, the main objectives of minimizing 3D TV and increasing the CNR, without affecting the blur in the z-direction, were achieved. Taking into account Fig. 3 and Fig. 4, filtered 3D TV values are smaller than the 3D TV value of unfiltered data for $\lambda$ values greater than 100. $\lambda$ works as a weighting factor in Eq. (3), which means, it should ensure that the fidelity term has enough weight to keep fundamental information in the filtered data. For 3D TV values achieved with $\lambda$ inferior to 100 , the fidelity term has not sufficient influence on the results as there is too much regularization and unwanted artifacts are introduced. Therefore, in both cases, only results obtained with $\lambda$ values greater than 100 were considered as valid. It is observed that both the algorithms presented the expected behavior for this problem, achieving a minimum value of $3 \mathrm{D} \mathrm{TV}$, with a significant reduction when compared with unfiltered data. The $2 \mathrm{D}$ minimization filter applied to all slices allowed a $25.57 \%$ decrease with $\lambda=197$, while the 3D filter achieved a $35.17 \%$ reduction in the $3 \mathrm{D}$ TV value with $\lambda=225$ (Table 2 ). As 3D TV considers alterations in $\mathrm{x}$, 
$\mathrm{y}$ and $\mathrm{z}$, the algorithm which is truly applied in $\mathrm{z}$ (taking into account adjacent slices Fig. 2(b)), results in the greatest reduction.

The $\lambda$ value which allowed the maximization of $\mathrm{CNR}$ at $90^{\circ}$ was the same for the sequentially applied 2D minimization filter (197), while for the 3D minimization it was changed to 195 (Fig. 5). With these $\lambda$ values, a significant improvement in CNR at $90^{\circ}$ was achieved: $30.84 \%$ and $36.47 \%$ for the $2 \mathrm{D}$ and 3D filters, respectively (Table 3 ). These quantitative values resulted in smoother rendered images in Fig. 7 (b1) and (c1) when compared with Fig. 7 (a1). Blur in z-direction, measured through FWHM of $0.5 \mathrm{~mm}$ and $5 \mathrm{~mm}$ disks at $90^{\circ}$, has not increased in any case, achieving a reduction of $6.28 \%$ for 5 mm disk when filtered with the 2D algorithm slice-by-slice (Table 3). This effect is visible through a slight decrease in disk thickness in Fig. 7(b1). For the $0.5 \mathrm{~mm}$ disk (Fig. 7 (a2) to (c2)), the differences are not visually evident (with variations of about $2 \%$ to $3 \%$ in FWHM). 3D TV minimization filter presented better results for the increase in CNR (and resulting noise reduction) but $2 \mathrm{D}$ minimization filter resulted in a greater reduction in $\mathrm{z}-$ blur. This is due to its slice-by-slice application, where it does not spread information between adjacent slices to make data smoother. Although the actual thickness of the disks is $1 \mathrm{~mm}$, both presented higher values (Table 3) and the larger the disk, the greater the contamination in z. Despite reducing this contamination, this type of filter does not solve this problem. Deconvolution methods may be a good option but are quite sensitive to noise. As these TV minimization filters showed a significant increase in CNR (reducing noise), a combination of both approaches can yield interesting results that should be studied in the future.

As pointed out in this paper, all data dimensions are important. In this way, it is essential to also ascertain image quality at $0^{\circ}$, i.e., in $x y$ (usual $2 \mathrm{D}$ visualization plane). From Table 4, we observe the results obtained at $0^{\circ}$ are consistent with those at $90^{\circ}$. Both 
filters avoid FWHM deterioration with a variation of about $0 \%$ for the smallest disk and $2.5 \%$ for the largest disk. The 2D minimization filter allowed a $19.48 \%$ increase in CNR, while 3D filter again showed a better result with an increase of $36.25 \%$ (similar to $90^{\circ}$ ). This improvement in CNR is quite visible in Fig. 6(a1) to (c1) through smoother images with a crescent degree from left (Fig. 6 (a1)) to right (Fig. 6 (c1)). The small variations in FWHM resulted in preservation of the edges and alterations not visible to naked eye in the results of Fig. 6, both for the larger disk (Fig. 6 (a1) to (c1)) and for the smallest (Fig. 6 (a2) to (c2)).

Considering clinical data, results obtained with $\lambda$ values above 70 were considered valid for both algorithms (Fig. 8 and Fig. 9). This lower threshold (when compared to phantom) varies according to $\sigma_{\mathrm{B}}$ of the unfiltered data. As this value is higher for clinical data (which contain much more anatomical information), the minimum value of $\lambda$ must be lower [42]. With $\lambda=135,2 \mathrm{D}$ filter achieved a $22.02 \%$ minimization on 3D TV. The 3D filter reached a 3D TV minimum (decrease of 31.29\%) with $\lambda=145$ (Table 5). Besides, according to Table 6 , the results of the metrics studied at $0^{\circ}$ and $90^{\circ}$ are in agreement with those obtained for the phantom. At $0^{\circ}$, the CNR has here a more significant increase: $39.09 \%$ and $49.43 \%$ for the $2 \mathrm{D}$ and $3 \mathrm{D}$ filters, respectively. At $90^{\circ}$, the variations are of a similar order of magnitude: $20.40 \%$ and $25.40 \%$ for each case, respectively. CNR measures in clinical data should be interpreted with caution because clinical background often considers anatomical noise, which may influence the results. On the other hand, taking into account the calcification in this case, also the variations obtained for FWHM are similar to those of the phantom. At $0^{\circ}$, these variations are numerically small $(\sim 0 \%)$ for both filters. At $90^{\circ}$, the $2 \mathrm{D}$ filter achieves once again a greater blur reduction $(5.55 \%)$ than 3D filter (3.69\%). Images from Fig. 10 are in accordance with these quantitative results. Areas of higher noise (clearly visible in the zoom of Fig. 10(a1) and (d1)) become 
much softer with the application of any of the filters (Fig. 10 (b1, c1, e1, f1). Calcification edges were preserved at $0^{\circ}$ and its spread was reduced at $90^{\circ}$ (Fig. $10(\mathrm{e} 1)$ ).

As mentioned before, different rendering parameters such as voxel size in $\mathrm{z}$, sample distance or transfer functions can influence the obtained results. Therefore, the presented results are compared only with each other or with results obtained under the same conditions. In addition to visualization parameters, also acquisition parameters such as $\mathrm{mAs}$ (which produces data with different noise levels) and $\mathrm{kVp}$ (which produces data with different contrast levels) can affect the results. Despite these limitations, the presented and validated methodologies are reproducible in other scenarios.

We think it is important to compare these results obtained after reconstruction with FBP with DBT data reconstructed with iterative algorithms, which have shown interesting results in terms of reducing the out-of-plane artifact.

\section{CONCLUSIONS}

To solve the same TV minimization problem in DBT data, two 3D filters were studied. The obtained results were analyzed at two angles $\left(0^{\circ}\right.$ and $\left.90^{\circ}\right)$ with a $3 \mathrm{D}$ visualization through volume rendering. The filters differ in their application. One considers a slice-by-slice optimization, sequentially traversing all slices of the data. The other considers the intensity values of adjacent slices to make this optimization on each voxel.

For the rendered phantom images, the suitable Lagrange multiplier $(\lambda)$ to be used in the discrete equation of each filter has been studied with two objectives: first, 3D TV minimization (validating the algorithms) and second, CNR maximization at $90^{\circ}$, without increasing the blur in z. Analysis at other visualization angles (such as at $45^{\circ}$ ) should be considered in future work. 
The performance of each filter was also tested with a clinical case containing a calcification. The results obtained for the phantom and the clinical data are very encouraging with a significantly increased CNR at $0^{\circ}$ and $90^{\circ}$ and a small reduction in blur at $90^{\circ}$ (slight reduction of the out-of-plane artifact). In addition, both approaches were applied in a considerably short time, taking into account the number of voxels in question.

This study presents the particularity of two 3D filters and analyzes the performance via 3D visualization. In this way, it is possible to get a sense of the impact of these algorithms on the data in a direct way, by visualizing the DBT data at once from several angles.

\section{ACKNOWLEDGMENTS}

This work was supported by Universidade de Lisboa (PhD grant) and Fundação para a Ciência e Tecnologia - Portugal (Grant No. SFRH/BD/135733/2018 and FCTIBEB Strategic Project UID/BIO/00645/2013).

\section{REFERENCES}

1. Ferlay, J., et al., Cancer incidence and mortality patterns in Europe: Estimates for 40 countries and 25 major cancers in 2018. European Journal of Cancer, 2018. 103: p. 356387.

2. Siegel, R.L., K.D. Miller, and A. Jemal, Cancer statistics, 2019. CA Cancer J Clin, 2019. 69(1): p. 7-34.

3. Berry, D.A., et al., Effect of screening and adjuvant therapy on mortality from breast cancer. N Engl J Med, 2005. 353(17): p. 1784-92.

4. Independent UK Panel on Breast Cancer Screening, The benefits and harms of breast cancer screening: an independent review. The Lancet, 2012. 380(9855): p. 1778-1786.

5. Food and Drug Administration (FDA) U.S. . Accreditation/Certification for Facilities Utilizing a DBT System with FFDM Images. 2011 [cited 2020 February ]; Available from: http://www.fda.gov/Radiation-

EmittingProducts/MammographyQualityStandardsActandProgram/FacilityCertificationand Inspection/ucm114148.htm.

6. Gennaro, G., et al., Digital breast tomosynthesis versus digital mammography: a clinical performance study. Eur Radiol, 2010. 20(7): p. 1545-1553. 
7. Brandt, K.R., et al., Can Digital Breast Tomosynthesis Replace Conventional Diagnostic Mammography Views for Screening Recalls Without Calcifications? A Comparison Study in a Simulated Clinical Setting. American Journal of Roentgenology, 2013. 200(2): p. 291298.

8. Bonafede, M.M., et al., Value analysis of digital breast tomosynthesis for breast cancer screening in a commercially-insured US population. ClinicoEconomics and Outcomes Research: CEOR, 2015. 7: p. 53-63.

9. Gao, Y., et al., Digital Breast Tomosynthesis Practice Patterns Following 2011 FDA Approval: A Survey of Breast Imaging Radiologists. Acad Radiol, 2017. 24(8): p. 947-953.

10. Destounis, S., A. Santacroce, and A. Arieno, DBT as a Screening Tool and a Diagnostic Tool. Current Breast Cancer Reports, 2017. 9(4): p. 264-271.

11. Sechopoulos, I., A review of breast tomosynthesis. Part I. The image acquisition process. Med Phys, 2013. 40(1): p. 014301.

12. Samala, R.K., et al., Digital breast tomosynthesis: Computer-aided detection of clustered microcalcifications on planar projection images. Phys Med Biol, 2014. 59(23): p. 74577477.

13. Good, W.F., et al., Digital breast tomosynthesis: a pilot observer study. AJR Am J Roentgenol, 2008. 190(4): p. 865-9.

14. Gur, D., et al., Digital breast tomosynthesis: observer performance study. AJR Am J Roentgenol, 2009. 193(2): p. 586-91.

15. Caumo, F., et al., Digital Breast Tomosynthesis with Synthesized Two-Dimensional Images versus Full-Field Digital Mammography for Population Screening: Outcomes from the Verona Screening Program. Radiology, 2018. 287(1): p. 37-46.

16. Benedikt, R.A., et al., Concurrent Computer-Aided Detection Improves Reading Time of Digital Breast Tomosynthesis and Maintains Interpretation Performance in a Multireader Multicase Study. American Journal of Roentgenology, 2017. 210(3): p. 685-694.

17. Balleyguier, C., et al., Improving digital breast tomosynthesis reading time: A pilot multireader, multi-case study using concurrent Computer-Aided Detection (CAD). Eur J Radiol, 2017. 97: p. 83-89.

18. Tchou, P.M., et al., Interpretation Time of Computer-aided Detection at Screening Mammography. Radiology, 2010. 257(1): p. 40-46.

19. Hofvind, S., et al., Digital Breast Tomosynthesis and Synthetic 2D Mammography versus Digital Mammography: Evaluation in a Population-based Screening Program. Radiology, 2018. 287(3): p. 787-794.

20. Simon, K., et al., Accuracy of Synthetic 2D Mammography Compared With Conventional 2D Digital Mammography Obtained With 3D Tomosynthesis. American Journal of Roentgenology, 2019. 212(6): p. 1406-1411.

21. van Schie, G., et al., Mass detection in reconstructed digital breast tomosynthesis volumes with a computer-aided detection system trained on 2D mammograms. Med Phys, 2013. 40(4): p. 041902.

22. Iotti, V., et al., Comparing two visualization protocols for tomosynthesis in screening: specificity and sensitivity of slabs versus planes plus slabs. Eur Radiol, 2019. 29(7): p. 3802-3811.

23. Petropoulos, A.E., et al., Quantitative assessment of microcalcification cluster image quality in digital breast tomosynthesis, 2-dimensional and synthetic mammography. Medical \& Biological Engineering \& Computing, 2020. 58(1): p. 187-209.

24. Venson, J.E., et al., A Case-Based Study with Radiologists Performing Diagnosis Tasks in Virtual Reality. Stud Health Technol Inform., 2017. 245: p. 244-248.

25. Suetens, P., Medical image analysis, in Fundamentals of Medical Imaging2009, Cambridge University Press: New York. p. 159-189.

26. O'Connell, A., et al., Cone-Beam CT for Breast Imaging: Radiation Dose, Breast Coverage, and Image Quality. American Journal of Roentgenology, 2010. 195(2): p. 496509.

27. Song, H., X. Cui, and F. Sun, Breast Tissue 3D Segmentation and Visualization on MRI. International Journal of Biomedical Imaging, 2013. 2013: p. 8. 
28. Jung, Y., et al., Occlusion and Slice-Based Volume Rendering Augmentation for PET-CT. IEEE Journal of Biomedical and Health Informatics, 2017. 21(4): p. 1005-1014.

29. Alyassin, A.M. Automatic transfer function generation for volume rendering of highresolution $x$-ray $3 D$ digital mammography images. in Medical Imaging 2002. 2002. SPIE.

30. Alyassin, A.M., et al., 3D Visualization of X-ray Tomosynthesis Digital Mammography Data: Preference Study, in Digital Mammography: IWDM 2002 - 6th International Workshop on Digital Mammography, H.-O. Peitgen, Editor 2003, Springer Berlin Heidelberg: Berlin, Heidelberg. p. 507-509.

31. Dharanija, R. and T. Rajalakshmi. A Conjunct Analysis for Breast Cancer Detection by Volume Rendering of Low Dosage Three Dimensional Mammogram. in Progress In Electromagnetics Research Symposium Proceedings. 2011. China.

32. Jerebko, A., et al. $3 D$ rendering methods for visualization of clusters of calcifications in digital breast tomosynthesis: a feasibility study. in ECR 2011. 2011. Vienna, Austria.

33. Neri, E., et al., The second ESGAR consensus statement on CT colonography. Eur Radiol, 2013. 23(3): p. 720-729.

34. Sidky, E.Y., et al., Enhanced imaging of microcalcifications in digital breast tomosynthesis through improved image-reconstruction algorithms. Med Phys, 2009. 36(11): p. 4920-32.

35. Ertas, M., et al., Digital breast tomosynthesis image reconstruction using $2 D$ and $3 D$ total variation minimization. Biomedical engineering online, 2013. 12: p. 112-112.

36. Seyyedi, S., et al., An object-oriented simulator for 3D digital breast tomosynthesis imaging system. Comput Math Methods Med, 2013. 2013: p. 250689-250689.

37. Michielsen, K., et al., Design of a model observer to evaluate calcification detectability in breast tomosynthesis and application to smoothing prior optimization. Med Phys, 2016. 43(12): p. 6577-6587.

38. Liu, Y., et al., An adaptive multiscale anisotropic diffusion regularized image reconstruction method for digital breast tomosynthesis. Australasian Physical \& Engineering Sciences in Medicine, 2018. 41(4): p. 993-1008.

39. Gomi, T. and Y. Koibuchi, Use of a Total Variation Minimization Iterative Reconstruction Algorithm to Evaluate Reduced Projections during Digital Breast Tomosynthesis. BioMed Research International, 2018. 2018: p. 5239082-5239082.

40. Garrett, J.W., et al., Reduced anatomical clutter in digital breast tomosynthesis with statistical iterative reconstruction. Med Phys, 2018. 45(5): p. 2009-2022.

41. Krammer, J., et al., Evaluation of a new image reconstruction method for digital breast tomosynthesis: effects on the visibility of breast lesions and breast density. The British Journal of Radiology, 2019. 92(1103).

42. Mota, A.M., et al., Total variation minimization filter for DBT imaging. Med Phys, 2015. 42(6Part1): p. 2827-2836.

43. Mota, A.M., et al. An iterative algorithm for Total Variation minimization in DBT imaging. in VipIMAGE 2015, . 2015. Tenerife, Spain: CRC Press.

44. Mota, A.M., et al. 3D Total Variation Minimization Filter for Breast Tomosynthesis Imaging. 2016. Malmo, Sweden: Springer International Publishing.

45. Siemens. MAMMOMAT Inspiration - Tomosynthesis Option. 2015 [cited 2020 February]; Available from: https://www.accessdata.fda.gov/cdrh_docs/pdf14/P140011c.pdf.

46. Schroeder, W., K. Martin, and B. Lorensen, The Visualization Toolkit: An Object-oriented Approach to 3D Graphics. 4rd ed2006, USA: Kitware.

47. VTK. Visualization Toolkit - VTK. 2020 [cited 2020 February]; Available from: http://www.vtk.org/.

48. VTK - Interpolators. Visualization Toolkit - VTK - Interpolators. [cited 2020 February]; Available from: https://vtk.org/Wiki/VTK/Image_Interpolators.

49. Rudin, L.I., S. Osher, and E. Fatemi, Nonlinear total variation based noise removal algorithms. Physica D: Nonlinear Phenomena, 1992. 60(1): p. 259-268.

50. Le, T., R. Chartrand, and T.J. Asaki, A Variational Approach to Reconstructing Images Corrupted by Poisson Noise. Journal of Mathematical Imaging and Vision, 2007. 27(3): p. 257-263. 
51. Sawatzky, A., et al. Total Variation Processing of Images with Poisson Statistics. 2009. Berlin, Heidelberg: Springer Berlin Heidelberg.

52. Rudin, L.I., S. Osher, and E. Fatemi, Nonlinear total variation based noise removal algorithms. Phys. D, 1992. 60(1-4): p. 259-268. 